\title{
Randomized comparison of awake nonresectional versus nonawake resectional lung volume reduction surgery
}

\author{
Eugenio Pompeo, MD, ${ }^{\mathrm{a}}$ Paola Rogliani, MD, ${ }^{\mathrm{b}}$ Federico Tacconi, MD, ${ }^{\mathrm{a}}$ Mario Dauri, MD, ${ }^{\mathrm{c}}$ \\ Cesare Saltini, MD, ${ }^{\mathrm{b}}$ Giuseppe Novelli, PhD, ${ }^{\mathrm{d}}$ and Tommaso C. Mineo, MD, ${ }^{\mathrm{a}}$ for the Awake Thoracic \\ Surgery Research Group
}

Objective: The study objective was to assess in a randomized controlled study (NCT00566839) the comparative results of awake nonresectional or nonawake resectional lung volume reduction surgery.

\begin{abstract}
Method: Sixty-three patients were randomly assigned by computer to receive unilateral video-assisted thoracic surgery lung volume reduction surgery by a nonresectional technique performed through epidural anesthesia in 32 awake patients (awake group) or the standard resectional technique performed through general anesthesia in 31 patients (control group). Primary outcomes were hospital stay and changes in forced expiratory volume in 1 second. During follow-up, the need of contralateral treatment because of loss of postoperative benefit was considered a failure event as death.
\end{abstract}

Results: Intergroup comparisons (awake vs control) showed no difference in gender, age, and body mass index. Hospital stay was shorter in the awake group (6 vs 7.5 days, $P=.04$ ) with 21 versus 10 patients discharged within 6 days $(P=.01)$. At 6 months, forced expiratory volume in 1 second improved significantly in both study groups $(0.28$ vs $0.29 \mathrm{~L})$ with no intergroup difference $(P=.79)$. In both groups, forced expiratory volume in 1 second improvements lasted more than 24 months. At 36 months, freedom from contralateral treatment was $55 \%$ versus $50 \%(P=.5)$ and survival was $81 \%$ versus $87 \%(P=.5)$.

Conclusions: In this randomized study, awake nonresectional lung volume reduction surgery resulted in significantly shorter hospital stay than the nonawake procedure. There were no differences between study groups in physiologic improvements, freedom from contralateral treatment, and survival. We speculate that compared with the nonawake procedure, awake lung volume reduction surgery can offer similar clinical benefit but a faster postoperative recovery. (J Thorac Cardiovasc Surg 2012;143:47-54)

\section{Supplemental material is available online.}

From the Departments of Thoracic Surgery, ${ }^{\mathrm{a}}$ Pneumology, ${ }^{\mathrm{b}}$ Anesthesiology, and Intensive Care, ${ }^{\mathrm{c}}$ and Biopathology and Diagnostic Imaging, ${ }^{\mathrm{d}}$ Policlinico Tor Vergata University, Rome, Italy.

Disclosures: Authors have nothing to disclose with regard to commercial support.

Read at the 91st Annual Meeting of The American Association of Thoracic Surgeons, Philadelphia, Pennsylvania, May, 7-11, 2011.

Awake Thoracic Surgery Research Group. Tor Vergata University, Rome, Italy: Eugenio Pompeo, MD (Principal Investigator), Maria Antonietta Ceccon, MD, Ludovica Celidonio, MD, Benedetto Cristino, MD, Maria Elena Cufari, MD, Mario Dauri, MD, Eleonora Fabbi, MD, Francesca Leonardis, MD, Luca Frasca, MD, Mario Marino, MD, Roberto Massa, MD, Tommaso Claudio Mineo, MD, Sarit Nahmias, MD, Giuseppe Novelli, PhD, Ilaria Onorati, MD, Augusto Orlandi, MD, Alessandra Picardi, MD, Paola Rogliani, MD, Alessandro Fabrizio Sabato, MD, Elisabetta Sabato, MD, Cesare Saltini, MD, Anna Maria Servadio, Federico Tacconi, MD, Gian Luca Vanni, MD. University La Sapienza, Rome, Italy: Italo Nofroni, BS.

Received for publication May 6, 2011; revisions received Aug 1, 2011; accepted for publication Sept 23, 2011; available ahead of print Nov 7, 2011.

Address for reprints: Eugenio Pompeo, MD, Cattedra di Chirurgia Toracica, Policlinico Università Tor Vergata, V.le Oxford, 81, 00133 Rome, Italy (E-mail: pompeo@med.uniroma2.it).

$0022-5223 / \$ 36.00$

Copyright (c) 2012 by The American Association for Thoracic Surgery

doi:10.1016/j.jtcvs.2011.09.050
Video clip is available online.

Lung volume reduction surgery (LVRS) is a wellestablished, palliative surgical treatment for severe emphysema that has shown to significantly improve pulmonary function, exercise capacity, quality of life, and survival in selected patients. ${ }^{1}$

Highly satisfactory clinical results have been reported by bilateral LVRS performed through median sternotomy ${ }^{2}$ and unilateral or bilateral LVRS performed through videoassisted thoracic surgery (VATS) ${ }^{3}$ or thoracotomy. ${ }^{4}$ Unfortunately, none of these approaches has minimized the significant morbidity and long hospitalization that are commonly associated with this surgical procedure. As a result, questions have been raised as to the cost-effectiveness of LVRS, eventually leading to a dramatic reduction in the number of procedures that are performed yearly. ${ }^{5}$ In an attempt of avoiding shortcomings of LVRS, novel surgical 


$$
\begin{aligned}
& \text { Abbreviations and Acronyms } \\
& \begin{aligned}
\mathrm{FEV} 1 & =\text { forced expiratory volume in } 1 \text { second } \\
\mathrm{FVC} & =\text { forced vital capacity } \\
\mathrm{LVRS} & =\text { lung volume reduction surgery } \\
\mathrm{PaCO}_{2} & =\text { arterial carbon dioxide tension } \\
\mathrm{PaO}_{2} / & =\text { ratio of arterial oxygen tension to } \\
\mathrm{FIO}_{2} & \text { fraction of inspired oxygen } \\
\mathrm{QR} & =\text { quartile range } \\
\mathrm{RV} & =\text { residual volume } \\
\mathrm{VATS} & =\text { video-assisted thoracic surgery } \\
\mathrm{WT} & =\text { walking test }
\end{aligned}
\end{aligned}
$$

and endoscopic ${ }^{6}$ lung volume reduction methods are being actively investigated.

Because morbidity can be negatively affected by both the need of general anesthesia and the deep resection of lung tissue in LVRS, we have developed an awake nonresectional method that respects the basic principles of resectional LVRS but adds theoretic advantages, including the possibility of being performed through sole thoracic epidural anesthesia in fully awake patients.

Preliminary findings from our group have suggested that awake nonresectional LVRS can be easily performed and optimally tolerated, and can offer clinical improvements that can be superior to those achieved by the standard resectional method. ${ }^{7}$ Moreover, in a recent study comparing nonresectional LVRS performed by awake or nonawake anesthesia, a shorter hospital stay and reduced costs were observed in the awake group. ${ }^{8}$

The current prospective randomized study comparatively assesses the outcomes of awake nonresectional versus nonawake resectional LVRS performed by unilateral VATS.

\section{MATERIAL AND METHODS}

Sixty-three patients undergoing unilateral LVRS by VATS at the Tor Vergata University School of Medicine between December 2002 and October 2006 were randomized to receive awake nonresectional (32 patients, awake group) or nonawake resectional (31 patients, control group) LVRS by VATS.

The study was approved by the Tor Vergata ethical committee, and all patients provided written informed consent. The main characteristics of the trial have been registered at the ClinicalTrials.gov site (trial No. NCT00566839).

Inclusion and exclusion criteria entailed findings of severe smokingrelated emphysema with upper-lobe predominance, associated with severe disability despite maximized medical care and no contraindication for VATS or thoracic epidural catheterization (Table E1).

Preoperative workup included spirometry with plethysmography, highresolution computed tomography of the chest, assessment of arterial blood gases, and assessment of diffusion capacity by the single breath technique.

\section{Study Design}

Results were assessed according to 2 different outcome domains: 1 surgical and 1 clinical.

\section{Surgical Domain}

The primary outcome measure was hospital stay. For this purpose, criteria for discharge were standardized. Removal of chest tubes was allowed when daily fluid drainage was less than $200 \mathrm{~mL}$ and air leaks stopped. In patients with minimal air leak and 1 chest tube remaining, a Heimlich valve was connected to the tube, and if this proved well tolerated by the patient and did not interfere with radiologically assessed complete lung expansion, discharge was allowed 24 hours later provided acceptance of a 48-hourbased outpatient clinical control program. Secondary outcome measures included 90-day mortality, ratio of arterial oxygen tension to fraction of inspired oxygen $\left(\mathrm{PaO}_{2} / \mathrm{FIO}_{2}\right)$, and arterial carbon dioxide tension $\left(\mathrm{PaCO}_{2}\right)$, assessed at 3 fixed time points $(\mathrm{T} 1=$ preoperative in lateral decubitus, $\mathrm{T} 2=$ end operative, and $\mathrm{T} 3=1$ hour after completion of the operation and weaning).

\section{Clinical Domain}

The primary outcome measure was forced expiratory volume in 1 second $\left(\mathrm{FEV}_{1}\right)$. The secondary outcome measures were plethysmographic residual volume (RV), forced vital capacity (FVC), exercise capacity assessed by both the 6-minute walking test (WT) and the maximal incremental treadmill test, dyspnea graded according to the Modified Medical Research Council scale, and health-related quality of life physical functioning domain scored according to the 36-Item Short Form questionnaire. All clinical measures were assessed at 6 months and every 6 months thereafter.

During follow-up, the need of contralateral treatment was considered a failure event as death. Contralateral LVRS was performed according to strict objective criteria, including loss of benefit in $\mathrm{FEV}_{1}$ or RV back to baseline values.

\section{Statistics}

We calculated that we would need to enroll 30 patients per group to have a statistical power of $80 \%$ with an alpha error of 0.05 to test the hypothesis that compared with nonawake resectional LVRS, the awake resectional procedure is associated with a $35 \%$ greater proportion of early discharges ( $\leq 6$ days). In regard to $\mathrm{FEV}_{1}$ improvement, 27 patients were calculated to be necessary to test the hypothesis of a greater postoperative $\mathrm{FEV}_{1}$ improvement at 6 months in the same group according to preliminary findings of a pilot study $\left(\Delta \mathrm{FEV}_{1}:+0.31 \mathrm{vs}+0.22 \mathrm{~L}\right)^{7}$

Randomization was carried out centrally by means of a computergenerated sequence of casual numbers in which treatment arms were assigned to paired and unpaired numbers, respectively.

Group descriptive statistics are presented as mean \pm standard deviation unless indicated otherwise. Two-way analysis of variance for repeated measures was used to evaluate surgical and clinical domain outcomes. Unpaired data were assessed stepwise by $t$ test and Mann-Whitney test as appropriate. Two-tailed Fisher exact test was used to compare proportions. Survival and freedom from contralateral treatment were assessed by the Kaplan-Meier method, and the log-rank test was used to match curves. Outcome analysis was carried out on an intention-to-treat basis. Statistical analysis was performed by Statistica software version 7.0 (StatSoft Inc, Tulsa, Okla).

\section{Anesthesia}

Awake anesthesia. Thoracic epidural catheterization was performed between $\mathrm{T} 4$ and $\mathrm{T} 5$ to achieve somatosensory and motor block between the $\mathrm{T} 1$ and T8 level while preserving diaphragmatic motion. In the operating room, patients received a continuous infusion of ropivacaine $0.5 \%$ and sufentanil $1.66 \mu \mathrm{g} / \mathrm{mL}$ into the epidural space. During the procedure, patients breathed oxygen through a Venturi facemask to keep oxygen saturation greater than $90 \%$. Permissive hypercapnia was accepted and did not require correction unless $\mathrm{pH}$ decreased to less than 7.2. During wound closure, the anesthetic regimen was changed to ropivacaine $0.16 \%$ and sufentanil $1 \mu \mathrm{g} / \mathrm{mL}$ at 2 to $5 \mathrm{~mL} / \mathrm{h}$. 
Nonawake anesthesia. After insertion of an epidural catheter between $\mathrm{T} 5$ and $\mathrm{T} 8$ for continuous infusion of ropivacaine, general anesthesia was induced by intravenous propofol (1.5-2 $\mathrm{mg} / \mathrm{kg})$, fentanyl $(0.1 \mathrm{mg})$, and vecuronium $(0.1 \mathrm{mg} / \mathrm{kg})$ and subsequently maintained using propofol, fentanyl, and vecuronium. Intraoperative ventilatory management and 1-lung ventilation were ensured by a left-sided double-lumen tube.

At the end of the operation (or after weaning if general anesthesia was used), all patients were transferred to the recovery room until cardiorespiratory conditions were considered stable by the anesthesiologist to allow direct return to the ward according to a fast-track policy. In all instances, the epidural catheter was removed 48 hours after the operation.

\section{Surgical Technique}

All surgical procedures were carried out by VATS through a 4-flexible trocar access, with a 30-degree 10-mm camera and standard instrumentation. Independently of the type of surgical method, the aim of the operation was to reduce the overall lung volume by approximately $20 \%$ to $30 \%$.

Awake nonresectional lung volume reduction surgery. Surgical pneumothorax was created by insertion of the first flexible trocar; if lung hyperinflation persisted despite the intrapleural atmospheric pressure environment, an EndoPaddle (Covidien, Norwalk, Conn) was gently applied onto the lung to minimize lung movements during spontaneous ventilation and improve surgical maneuvering, particularly when dealing with pleural adhesions, which could be easily cut whenever necessary. The most severely destroyed target areas were identified and pushed downward while redundant lung edges were grasped by 2 ring forceps. Subsequently, both lung edges were grasped together by 1 ring forceps and a 45-mm, non-cutting endostapler (Endopath 45NK; Ethicon Endosurgery, Pomezia, Italy) was fired on the plicated lung region starting at the lung apex and continuing by firing 2 other cartridges, 1 ventrally and 1 dorsally to perform a linear, interrupted suture and reducing the lung volume by $20 \%$ to $30 \%$ (Video E1).

Nonawake resectional lung volume reduction surgery. After insertion of the camera and careful exploration of the lung, the most severely emphysematous lung regions were grasped by 2 ring forceps, and staple excision of a reversed hockey stick-like single strip of lung tissue was carried out to reduce the overall lung volume by $20 \%$ to $30 \%$. In both groups, 2 chest tubes were inserted at the end of the procedure.

\section{RESULTS}

During the study period, of 87 eligible subjects, 24 patients refused randomization, whereas intraoperatively, 3 patients required conversion to general anesthesia ( 2 patients in the awake group) or thoracotomy (1 patient in the control group).

The mean age in the awake and control groups was $64 \pm$ 9 years and $65 \pm 7$ years, respectively $(P=.50)$. Study groups were relatively well matched in main baseline data (Table 1).

\section{Surgical Outcome Domain}

Primary outcome. Median hospital stay was 6 days (quartile range [QR], 3.0 days) in the awake group and 7.5 days ( $\mathrm{QR}, 4.0$ days) in the control group $(P=.04)$. Twenty-one patients $(66 \%)$ in the awake group and 10 patients $(32 \%)$ in the nonawake group were discharged within 6 days $(P=.01$, Figure 1). Four and 7 patients were discharged with a Heimlich valve in the awake and control groups, respectively $(P=.51)$.
TABLE 1. Preoperative data

\begin{tabular}{|c|c|c|c|}
\hline & $\begin{array}{c}\text { Awake group } \\
\text { Mean } \pm \text { SD }\end{array}$ & $\begin{array}{c}\text { Control group } \\
\text { Mean } \pm \text { SD }\end{array}$ & $\begin{array}{c}\text { Intergroup } \\
P \text { value } \\
\end{array}$ \\
\hline BMI & $24 \pm 4$ & $23 \pm 3$ & .16 \\
\hline $\mathrm{FEV}_{1}(\mathrm{~L})$ & $0.82 \pm 0.3$ & $0.78 \pm 0.2$ & .47 \\
\hline $\mathrm{FEV}_{1}(\%)$ & $29 \pm 9$ & $27 \pm 7$ & .32 \\
\hline FVC (L) & $2.36 \pm 0.8$ & $2.35 \pm 0.6$ & .94 \\
\hline $\mathrm{FVC}(\%)$ & $64 \pm 17$ & $63 \pm 16$ & .84 \\
\hline $\mathrm{RV}(\mathrm{L})$ & $5.05 \pm 1.0$ & $5.29 \pm 0.7$ & .31 \\
\hline RV (\%) & $217 \pm 40$ & $229 \pm 38$ & .24 \\
\hline TLC (L) & $8.17 \pm 1.5$ & $8.81 \pm 0.9$ & .05 \\
\hline $\operatorname{TLC}(\%)$ & $129 \pm 17$ & $140 \pm 17$ & .03 \\
\hline 6-minute WT (m) & $300 \pm 112$ & $329 \pm 98$ & .29 \\
\hline MITT (Bruce class) & $0.58 \pm 0.3$ & $0.65 \pm 0.4$ & .38 \\
\hline $\mathrm{PaO}_{2}(\mathrm{~mm} \mathrm{Hg})$ & $68 \pm 8.0$ & $67 \pm 6.0$ & .37 \\
\hline $\mathrm{PaCO}_{2}(\mathrm{~mm} \mathrm{Hg})$ & $41 \pm 5.0$ & $41 \pm 3.0$ & .69 \\
\hline Dyspnea index (score) & $3.5 \pm 0.6$ & $3.4 \pm 0.6$ & .41 \\
\hline PF (36-Item Short Form) & $28 \pm 13$ & $29 \pm 13$ & 68 \\
\hline
\end{tabular}

BMI, Body mass index; MITT, maximal incremental treadmill test; dyspnea index, modified Medical Research Council dyspnea score; $T L C$, total lung capacity; $P F$, physical functioning.

Secondary outcomes. Intraoperative change in oxygenation variables is shown in Figure 2, which shows that $\mathrm{PaO}_{2} / \mathrm{FIO}_{2}$ was reduced at $\mathrm{T} 2$ in both groups but returned toward the preoperative value at T3 in the awake group only, whereas it continued to deteriorate after weaning in the control group.

$\mathrm{PaCO}_{2}$ also behaved differently between the study groups. In the awake group, a higher value of permissive hypercapnia developed at $\mathrm{T} 2$, although this rapidly decreased toward the preoperative value at T3; in the control group, $\mathrm{PaCO}_{2}$ remained at a lower level than in the awake group at $\mathrm{T} 2$ but increased thereafter.

No patient in either group required intensive care unit stay, whereas time spent in the recovery room was significantly longer in the nonawake group $(228 \pm 68$ minutes vs $93 \pm 43$ minutes, $P<.00001$ ).

The nonawake group had only 1 operative mortality $(P=1.0)$. Nonfatal adverse events occurred in 7 patients in the awake group ( 6 prolonged air leaks and 1 atrial fibrillation) and 16 patients in the nonawake group (15 prolonged air leaks, 3 atrial fibrillations, and 1 pneumonia; $P=.019)$. Three patients had more than 1 complication.

\section{Clinical Outcome Domain}

No patient was lost to follow-up.

Primary outcome. At 6 months, median $\triangle \mathrm{FEV}_{1}$ was 0.28 $\mathrm{L}(\mathrm{QR}: 0.16 \mathrm{~L})$ in the awake $(P<.00001)$ group versus 0.29 L (QR: $0.12 \mathrm{~L})$ in the control group $(P<.00001)$, with no intergroup difference $P=.81$ ) (Figure 1).

Secondary outcomes. Significant clinical improvements occurred in FVC, RV, WT, maximal incremental treadmill test, physical functioning, dyspnea index, and body mass index at 6 months. On awake versus nonawake group 

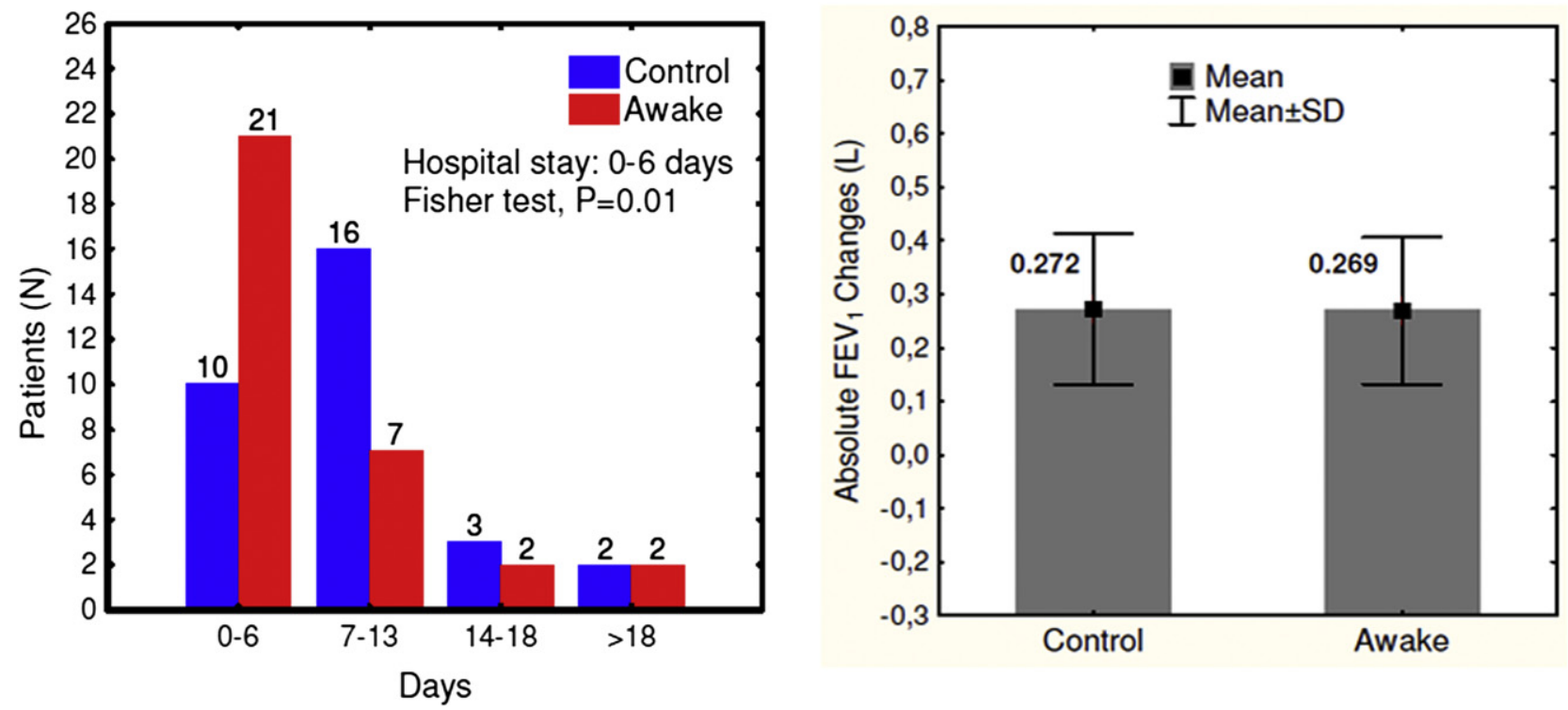

FIGURE 1. Outcome of primary end points in the study groups. Bar graph showing distribution of categorized hospital stay $(l e f t)$ and changes in FEV ${ }_{1}$ at 6 months (right).

comparisons, $\triangle \mathrm{FVC}$ was $0.40 \pm 0.3 \mathrm{~L}$ versus $0.35 \pm 0.2 \mathrm{~L}$ $(P=.42) ; \Delta \mathrm{RV},-0.91 \pm 0.4 \mathrm{~L}$ versus $-0.90 \pm 0.5 \mathrm{~L}$ $(P=.95) ; \Delta$ dyspnea index, $-1.34 \pm 0.6$ versus $-1.17 \pm 0.6$ $(P=.28)$; and $\Delta \mathrm{WT}, 81 \pm 51 \mathrm{~m}$ versus $85 \pm 37 \mathrm{~m}(P=.75)$.

Most clinical improvements observed at 6 months lasted for more than 2 years (Figure 3). At 24 months, 15 patients $(54 \%)$ had an $\triangle \mathrm{FEV}_{1}$ greater than $0.1 \mathrm{~L}$ in the awake group versus 17 patients $(63 \%)$ in the control group $(P=.48)$. At the 12- and 24-month assessments, significant betweengroup differences were observed in body mass index
$(P<.009)$ and percent predicted total lung capacity $(P=.03)$ (Table E2).

There were no differences between study arms in estimated rate of freedom from contralateral treatment $(55 \%$ vs $50 \%, P=.7)$ and survival $(81 \%$ vs $87 \%, P=.5)$ at 36 months (Figure 4). During follow-up, 12 and 14 patients required contralateral LVRS within 36 months in the awake and nonawake groups, respectively. Contralateral LVRS was performed because of deterioration of $\mathrm{FEV}_{1}$ in 18 patients and of RV in 8 patients with no 90-day mortality.
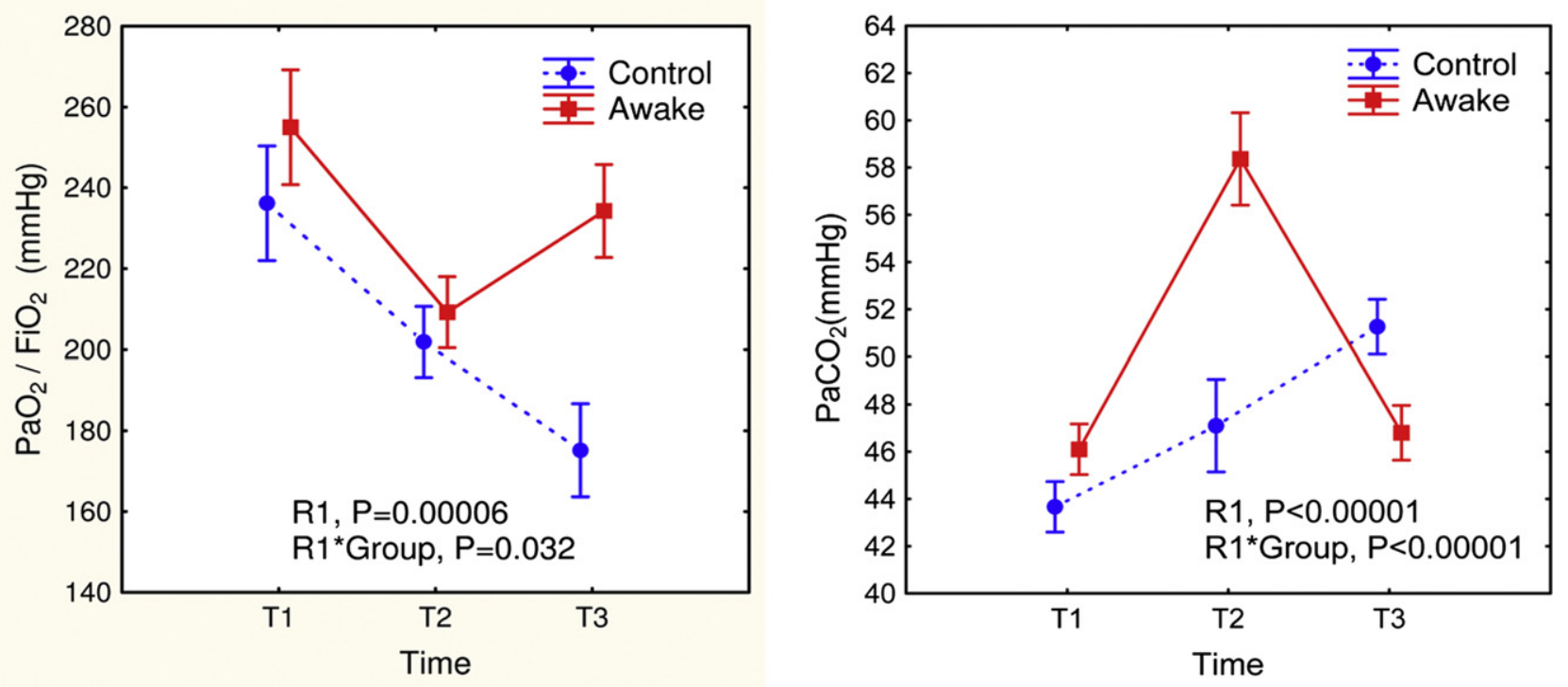

FIGURE 2. Perioperative behavior of $\mathrm{PaO}_{2} / \mathrm{FiO}_{2}$ (left) and $\mathrm{PaCO}_{2}$ (right) at 3 fixed time points (T1-T3). Analysis of variance: $\mathrm{R} 1=$ overall effect; $\mathrm{R} 1$ *group $=$ intergroup effect. 

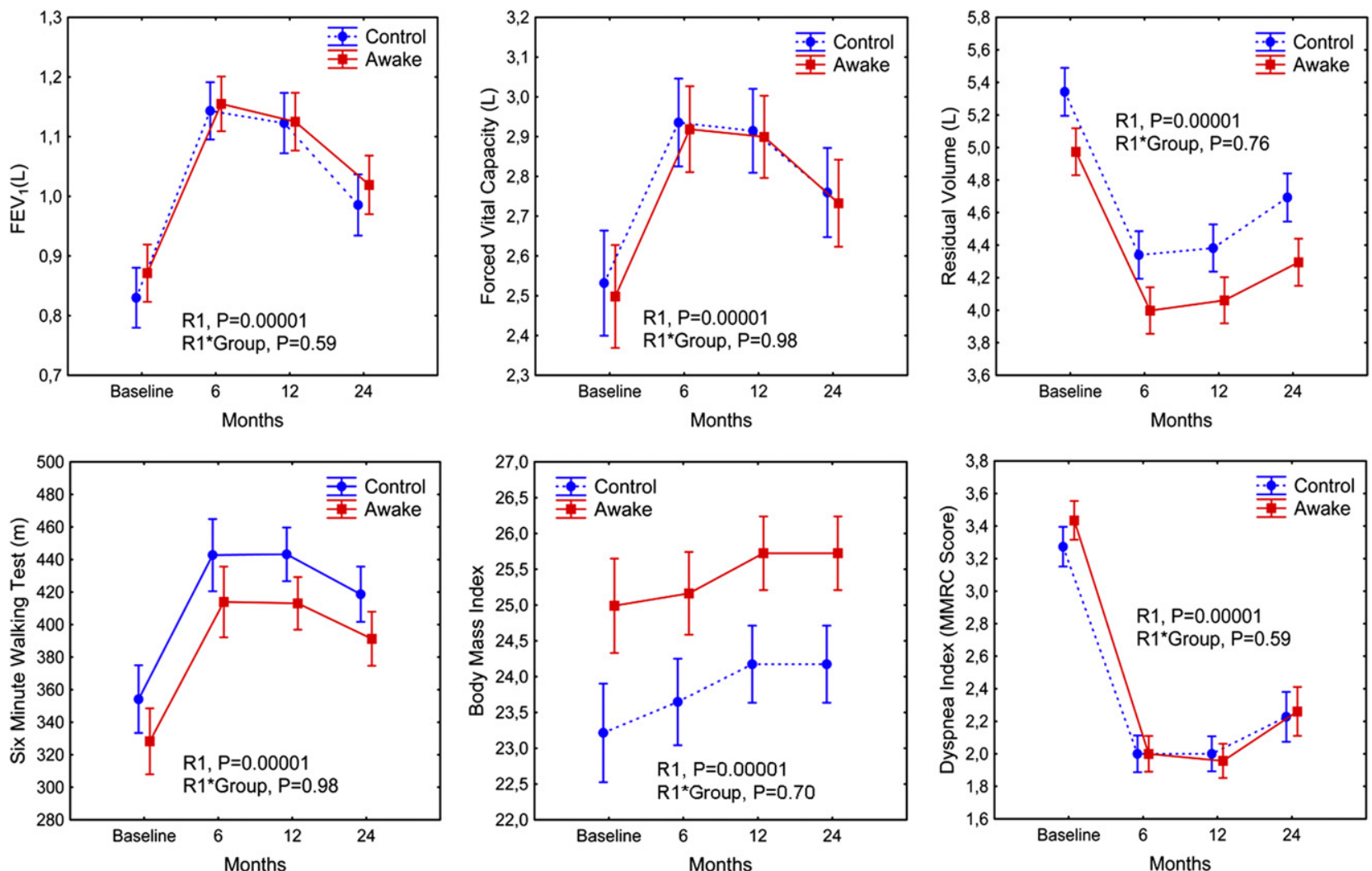

FIGURE 3. Pre- to postoperative changes in $\mathrm{FEV}_{1}$ (left upper), FVC (mid upper), RV (right upper), 6-minute WT (left lower), body mass index (mid lower), and dyspnea index (right lower).
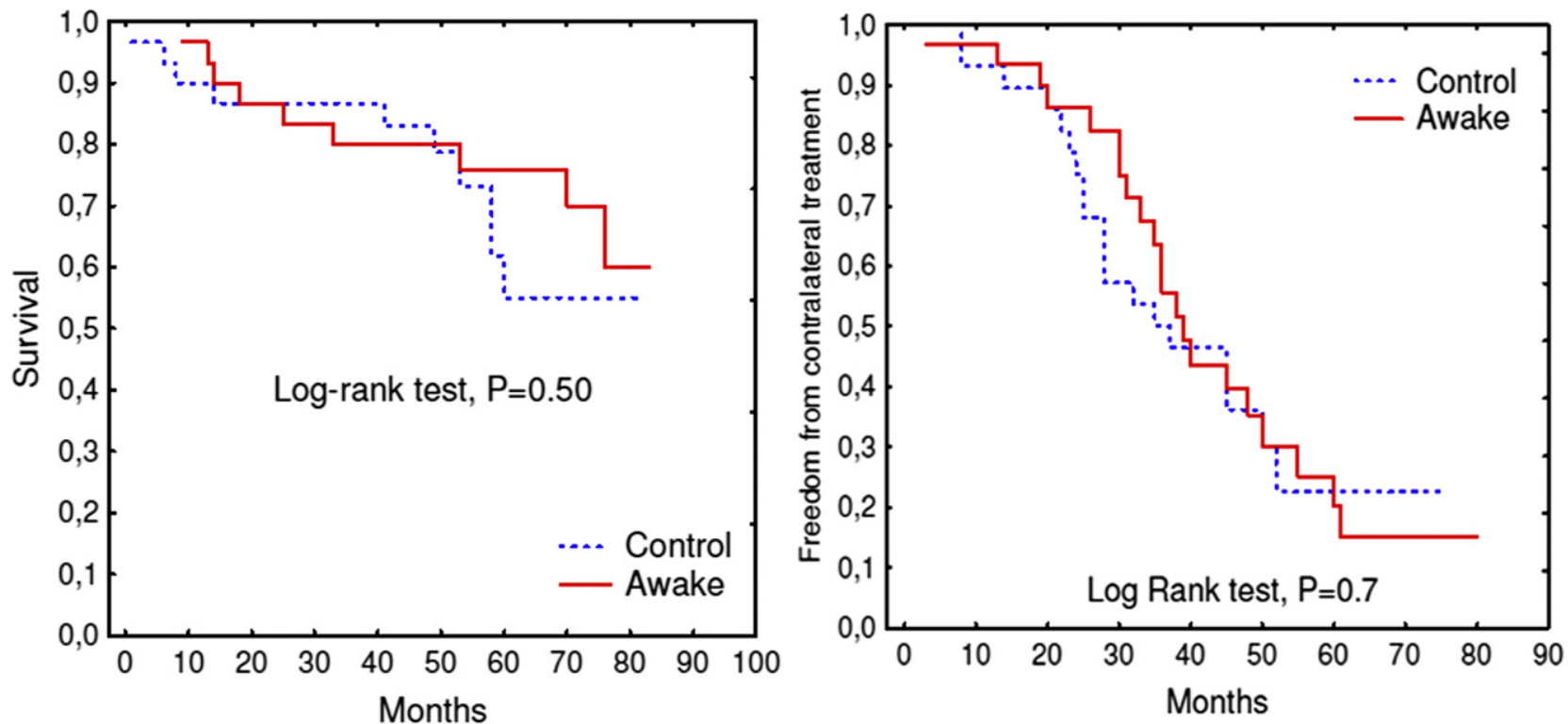

\begin{tabular}{|l|l|l|l|l|}
\hline Months & 12 & 24 & 36 & 48 \\
\hline Awake (N) & 31 & 28 & 26 & 24 \\
\hline Control (N) & 28 & 27 & 27 & 21 \\
\hline
\end{tabular}

\begin{tabular}{|l|c|c|c|c|}
\hline Months & 12 & 24 & 36 & 48 \\
\hline Awake (N) & 29 & 22 & 14 & 11 \\
\hline Control (N) & 26 & 19 & 14 & 9 \\
\hline
\end{tabular}

FIGURE 4. Kaplan-Meier curves of estimated survival (left) and freedom from contralateral treatment (right). Patients who were alive (left) and free from contralateral treatment (right) during follow-up are shown below each plot. 


\section{DISCUSSION}

General anesthesia and single-lung ventilation are deemed mandatory for LVRS, although it can be associated with risks of pneumonia, cardiovascular and neuromuscular adverse events, bronchospasm and airways injury, and a multifactorial lung injury related to mechanical ventilation. ${ }^{7}$

Most of the aforementioned adverse effects can be avoided by using thoracic epidural anesthesia in awake patients even though physiologic effects of such an unconventional choice are still not thoroughly investigated.

In this randomized study, awake nonresectional LVRS resulted in a shorter hospital stay and a significantly greater proportion of early discharges than the control group with significant and as yet equivalent 6-month improvements in $\mathrm{FEV}_{1}$. Significant improvements lasting more than 2 years also occurred in secondary clinical outcomes, including FVC, RV, WT, and subjective dyspnea with no intergroup difference. Furthermore, no difference was detected between study groups in survival and freedom from contralateral treatment at 36 months.

Nonresectional LVRS entailing fold plication of emphysematous lung regions has been anecdotally reported. ${ }^{9,10}$ Our nonresectional method has been specifically developed by one of the authors (E.P.) to be easily and safely performed in awake, spontaneously ventilating patients. The method differs from the others by entailing an introflexive plication of emphysematous lung tissue. Although the method respects the basic concepts advocated for resectional LVRS ${ }^{2}$ that include a reduction of $20 \%$ to $30 \%$ of the lung volume and stapled suture along a single ideal line, it adds some technical differences such as peripheral suturing to minimize interruption of segmental vessels and bronchi, an interrupted suture line, which is more flexible to favor reexpansion of the remodeled lung, and avoidance of discontinuation of visceral pleura with a 4-fold pleural buttress of the suture to minimize risk of air leaks. The validity of these theoretic advantages need to be proved experimentally.

\section{Surgical Domain Outcome}

The results of the current study showed that after awake nonresectional LVRS, $66 \%$ of patients were discharged within 6 days, whereas this was possible in only $32 \%$ of patients in the control group. It is difficult to discern whether this finding is to be attributed to the avoidance of general anesthesia and mechanical ventilation or to the use of a nonresectional method, although it is conceivable that both components played a role. In this respect, in a recent nonrandomized study comparing nonresectional LVRS performed by awake or nonawake anesthesia, median hospital stay and overall costs were reduced in the awake group with no intergroup difference in late clinical outcome. ${ }^{8}$ In the current study, results of the awake group compare favorably with previous reports of unilateral LVRS in which hospital stay ranged from 8 days ${ }^{4}$ to 17 days. ${ }^{11}$

Another issue regards our surgical strategy of unilateral treatment followed by contralateral LVRS delayed until benefits of the first operation are weaned. Despite the lack of randomized studies in this setting, findings of postoperative annual decay in $\mathrm{FEV}_{1}$ averaging $107 \mathrm{~mL}$ in unilateral LVRS and $250 \mathrm{~mL}$ in bilateral LVRS $^{3}$ suggest a role for a staged unilateral approach, which is now our standard strategy of treatment irrespective of the emphysema characteristics or the type of anesthesia that is chosen.

Perioperatively, the behavior of $\mathrm{PaO}_{2} / \mathrm{FIO}_{2}$ differed significantly among study groups with a progressive decrease between $\mathrm{T} 2$ and $\mathrm{T} 3$ in the control group and an initial reduction followed by a return toward the baseline value in the awake group. In a similar manner, $\mathrm{PaCO}_{2}$ increased to a greater extent in awake patients at $\mathrm{T} 2$ and returned toward baseline values at T3, whereas it progressively increased during time in the nonawake group. In accordance with these findings, Buduhan and coworkers ${ }^{12}$ demonstrated in an experimental study that there was an impairment in lung mechanical properties due to decreased respiratory compliance and increased gas trapping after nonawake LVRS. In the clinical setting, immediately after weaning, expiratory flow limitation hampers expiration and worsens gas trapping, leading to rapid shallow breathing that in time increases inspiratory workload, decreases respiratory muscle capacity, and hampers carbon dioxide elimination. Post-intubation airways irritation and accumulation of secretions, expiratory muscle recruitment, and occult small airway disease secondary to increased lung water are other factors that can increase gas trapping after nonawake LVRS. ${ }^{13}$ On the other hand, after awake LVRS, the maintained diaphragmatic motion facilitates a more physiologic ventilation in the early postoperative period.

\section{Clinical Domain Outcome}

At 6 months, $\mathrm{FEV}_{1}$ improved significantly in both study groups with no intergroup difference. These results are similar to those observed by other investigators with unilateral and bilateral resectional LVRS. ${ }^{4,11,14}$

Overall, significant improvements in respiratory function, exercise capacity, perceived quality of life, and subjective dyspnea were observed for more than 24 months in both study groups. These findings suggest that the combination of awake anesthesia and nonresectional LVRS did not jeopardize the possibility of achieving a meaningful reduction in lung volume and that postoperative beneficial effects did not deteriorate in the awake group more rapidly than those of the resectional method.

In a recent update of the National Emphysema Treatment Trial results, ${ }^{15}$ patients with upper-lobe predominant emphysema and low exercise capacity demonstrated improved 
3-year exercise capacity, 5-year relief of dyspnea, and better 5 -year survival than medically treated patients.

In the current series entailing all patients with upper-lobe emphysema, estimated 3-year survival was greater than $80 \%$ in both study groups. So far, endoscopic procedures proposed as an alternative to LVRS did not demonstrate comparable efficacy. ${ }^{6}$ Thus, we confide that results of our trial will provide further scientific data directed at accentuating the positive ${ }^{16}$ role of surgery in the optimal management of patients with emphysema.

\section{Limitations}

Limitations of this study include the small cohort with an intergroup difference in baseline total lung capacity and the mixed combination of 2 types of anesthesia and 2 types of surgical methods that negated the possibility to weigh the role of each single component in determining the final outcomes. To attenuate these concerns, we have completed a small, preliminary study comparing nonresectional LVRS performed by awake or nonawake anesthesia. ${ }^{8}$ However, we considered it unethical to perform a randomized study comparing the results of an unconventional LVRS method (eg, our nonresectional LVRS technique carried out by awake or nonawake anesthesia) because this surgical technique is currently used by our center only, and so far there were no other prospectively collected data confirming its reliability when compared with resectional LVRS, which is still considered the gold standard by most thoracic surgeons.

We acknowledge that both the design of the trial and our strategy entailing an intentional, initial unilateral operation may have led to an apparent overestimation of some outcome data because of exclusion of patients undergoing contralateral treatment during time. However, we were interested in comparing the degree of improvements in homogeneous cohorts including only patients treated in unilateral fashion. Nonetheless, we have considered the need of contralateral treatment a negative event as death, and assessed this secondary outcome measure by the KaplanMeier method, thus providing useful comparative data on long-term outcome of all patients.

\section{CONCLUSIONS}

In this randomized study, awake nonresectional LVRS was associated with a greater proportion of early discharges than the control group, while offering equivalent and longlasting clinical improvements in $\mathrm{FEV}_{1}$ and other outcome measures. In addition, no difference was observed between study groups in survival and need of contralateral treatment for up to 36 months.

\section{References}

1. National Emphysema Treatment Trial Research Group. A randomized trial comparing lung-volume-reduction surgery with medical therapy for severe emphysema. N Engl J Med. 2003;248:2059-73.
2. Cooper JD, Trulock EP, Triantafillou AN, Patterson GA, Pohl MS, Deloney PA, et al. Bilateral pneumectomy (volume reduction) for chronic obstructive pulmonary disease. J Thorac Cardiovasc Surg. 1995;109:106-19.

3. Brenner M, McKenna RJ Jr, Gelb AF, Fischel RJ, Wilson A. Rate of FEV1 change following lung volume reduction surgery. Chest. 1998;113:652-9.

4. Meyers BF, Sultan PK, Guthrie TJ, Lefrak SS, Davis GE, Patterson GA, et al. Outcomes after unilateral lung volume reduction. Ann Thorac Surg. 2008;86:204-12.

5. DeCamp M, McKenna RJ, Deschamps CC, Krasna MJ. Lung volume reduction surgery. Technique, operative mortality and morbidity. Proc Am Thorac Soc. 2008;5:442-6.

6. Sciurba FC, Ernst A, Herth FJ, Strange C, Criner GJ, Marquette CH, et al. A randomized study of endobronchial valves for advanced emphysema. N Engl J Med. 2010;363:1233-44

7. Mineo TC, Pompeo E, Mineo D, Tacconi F, Marino M, Sabato AF. Awake nonresectional lung volume reduction surgery. Ann Surg. 2006;243:131-6.

8. Pompeo E, Tacconi F, Mineo TC. Comparative results of non-resectional lung volume reduction performed by awake or non-awake anesthesia. Eur J Cardiothorac Surg. 2011;39:e51-8.

9. Swanson SJ, Mentzer SJ, DeCamp MM, Bueno R, Richard WG, Ingenito EP, et al. No-cut thoracoscopic lung plication: a new technique for lung volume reduction surgery. J Am Coll Surg. 1997;185:25-32.

10. Iwasaki M, Nishiumi N, Kaga K, Kanazawa M, Kuwamira I, Inoue H. Application of the fold plication method for unilateral lung volume reduction in pulmonary emphysema. Ann Thorac Surg. 1999;67:815-7.

11. Keenan RJ, Landreneau RJ, Sciurba FC, Ferson PF, Holbert JM, Brown ML, et al Unilateral thoracoscopic surgical approach for diffuse emphysema. $J$ Thorac Cardiovasc Surg. 1996;111:308-16.

12. Buduhan G, Tan L, Kasian K, Mink N. Volume reduction surgery impairs immediate postoperative pulmonary function in canine emphysema. Am J Respir Crit Care Med. 2006;174:1310-8.

13. Rivera L, Weissman C. Dynamic ventilator characteristics during weaning in postoperative critically ill patients. Anesth Analg. 1997;84:1250-5.

14. Ginsburg ME, Thomashow BM, Yip CK, DiMango AM, Maxfield RA, Bartels MN, et al. Lung volume reduction surgery using the NETT selection criteria. Ann Thorac Surg. 2011;91:1556-60.

15. NaunheimKS Wood DE, Moshenifar Z, Stenberg AL, Criner GJ, DeCamp MM, et al. National Emphysema Treatment Trial Research Group. Long-term followup of patients receiving lung-volume-reduction surgery versus medical therapy for severe emphysema by the National Emphysema Treatment Trial Research Group. Ann Thorac Surg. 2006;82:431-43.

16. Sanchez PG, Kucharczuk JC, Su S, Kaiser LR, Cooper JD. National Emphysema Treatment Trial redux: accentuating the positive. J Thorac Cardiovasc Surg. 2010;140:564-72.

\section{Discussion}

Dr Bryan Meyers (St Louis, Mo). The authors have provided us with a noteworthy trial comparing 2 alternative strategies for LVRS in patients with advanced emphysema. The degree of impairment in these patients is consistent with that seen in other reports, and the amount of $\mathrm{FEV}_{1}$ improvement of approximately $37 \%$ at 6 months is certainly comparable or even better than often seen with unilateral volume reduction surgery. So the clinical care is to be commended. The study is prospective and randomized, which is certainly what caught the attention of the program committee. I commend the authors on the ability to initiate and complete a prospective randomized study, particularly when the 2 arms of therapy are as disparate as awake versus nonawake anesthetic. That's quite a challenge, and I commend you. I do have a few concerns that you have already mentioned in your limitations that I want to underscore. The one problem I had was that the design changes 2 things as you move from one group to the other. You have awake and nonresection compared with nonawake and resection, and that leaves us in some doubt as we interpret the results of the study as to which factors might contribute to the overall end result. Why did you do that? Why not alter one thing at a time and 
then give us some insight as to how each factor might affect the outcome?

Dr Pompeo. This is one limitation of the study that we had considered. For this reason, we had performed a small prospective nonrandomized study comparing awake nonresectional lung volume reduction with nonawake nonresectional lung volume reduction. We recently published it in the European Journal of Cardiothoracic Surgery, and we specifically wanted to publish it before the presentation of this randomized study for this reason, because we were interested in seeing if the nonresectional method per se is sufficient to say that it can improve early results. What we have seen is that the results were not as good as with the double association of the nonresectional method and awake anesthesia. This might be one answer.

Dr Meyers. Another point I would make is that you analyzed this per protocol, that is, you chose not to use an intention-to-treat analysis. You excluded 2 patients who could not tolerate the awake procedure and 1 patient who was converted from the VATS approach to an open approach, and when we evaluate treatment strategies, we have to compare all patients who were exposed to that strategy and not just patients who were successfully treated with that strategy. So I would just point that out. The final point is that your sample size of 30 in each arm is small, although nowadays there aren't a whole lot of patients needing lung volume reduction, and it might be small by necessity. But you justified it by picking a primary outcome measure that I would argue is not a clinically important factor. The fraction of patients who are discharged at or before 6 days is not what I think of as the main thing that I want to evaluate when I compare 2 strategies for LVRS. So I'm not sure that the sample size was really sufficient to give us the full insight into these 2 therapies.

Dr Pompeo. This is probably true. We had to choose the outcome measure as a compromise to reach a sufficient power to perform the study. Obviously a larger sample size might have been better, but it's difficult when doing a single-center study to have such a large quantity of patients to randomize.

Dr Meyers. I would summarize by saying this was excellent clinical care. Both patients in both arms got a great boost with a unilateral LVRS, although my own bias would be to offer a bilateral operation, and the conduct of the trial could have been better, but it was thought provoking and novel therapy on the intervention arm.

Dr Pompeo. Thank you.

Dr Scott Swanson (Boston, Mass). I enjoyed that article and am interested in that technique. We actually published on that in the mid-1990s. Could you give us some tips on how you actually do it, particularly on an awake patient? Is there anything we can learn from you about the technical part of this operation?

Dr Pompeo. There are several small tricks you can apply to operate on these patients. What we have seen during time with this kind of operation is that if the operation becomes too long, permissive hypercapnia can become a problem for the patient. We have resolved this problem in the last years by inserting a chest tube at the beginning of the operation. In this way we can temporarily close the trocars with fingers or gauzes and allow reexpansion of the lung during the operation, and this has consistently reduced the $\mathrm{PaCO}_{2}$, during the procedure. Another thing is to discuss with the patient during the operation that difficulty in breathing can be due to the surgical pneumothorax, because the patient can feel difficulty even though the oxygenation remains at a satisfactory level. With experience you will find that the operation is quick, simple, and safe, and I think for these patients, avoiding general anesthesia is a big advance.

Dr J. Shrager. Scott, is this technique exactly what you described when you reported it? I seem to recall that you do some sort of twisting of the parenchyma that is a bit different than this?

Dr Swanson. I'm not quite sure. Yes, we were folding along and then stapling.

Dr Pompeo. But there is a difference between the techniques. Dr Swanson proposed a folding technique, repeatedly folding on the lung surface, whereas we perform a single introflexing plication. We push the lung down inside the underlying tissue and suture it above, along a single ideal line, as with the resectional technique.

Dr Shrager. Sort of like plicating a diaphragm. Is that what you're trying to describe?

Dr Pompeo. Yes, something like that.

Dr Swanson. I have just one follow-up question. Were you able to deal with adhesions through this technique, or was that a problem?

Dr Pompeo. Sorry?

Dr Swanson. Did you run into any adhesions in the chest during the awake cases, and if so, can you handle adhesions to the chest wall with this technique?

Dr Pompeo. Well, regarding adhesions, it's not really a problem. We normally use an EndoPaddle to push on the lung and see the apical parts of the lung better to cut the adhesions. It may require some more time than with a resectional technique, but it's quite simple as well.

Dr Walter Weder (Zurich, Switzerland). Congratulations on your work. I have 2 questions. Why did you offer all these patients a unilateral and not a bilateral volume reduction?

Dr Pompeo. We actually offered a staged bilateral procedure. What we do is initially perform a unilateral procedure. As you probably know, we are convinced that a staged bilateral procedure is better than a 1-stage bilateral procedure. Some studies have demonstrated that after a 1-stage bilateral procedure, the decay in $\mathrm{FEV}_{1}$ is approximately $250 \mathrm{~mL} /$ year, whereas after a unilateral procedure, the decay is approximately $107 \mathrm{~mL} / \mathrm{year}$, and we believe this is a good reason to do a staged procedure if the patient can improve significantly with a unilateral lung volume reduction.

Dr Weder. In your Abstract you stated that the improvement of $\mathrm{FEV}_{1}$ lasted for 3 years, and in your presentation you said it was statistically improved for 2 years. You excluded all patients who had failures and needed a second operation. Do you think it is correct to exclude the worst cases for evaluating the long-term effect?

Dr Pompeo. This is an important question. Our aim was not to demonstrate that lung volume reduction is capable of improving pulmonary function. We know that this is possible, and I think there are already enough data to demonstrate this. What we were interested in was to compare the results between the awake and nonawake procedures. I know that in this way, by excluding patients who were operated contralaterally, we have created a bias, but the bias is unavoidable, because otherwise we had to consider all the patients, and we had some patients operated unilaterally and some bilaterally. We would prefer to show the results of the bilateral procedures later. 
TABLE E1. Inclusion and exclusion criteria adopted for the trial

\section{Inclusion criteria}

Severe smoking-related emphysema with upper-lobe predominance

Severe disability despite maximized medical therapy including respiratory rehabilitation with Modified Medical Research Council dyspnea grade $\geq 3$

No clinically significant sputum production, bronchiectasis, or asthma

Post-bronchodilator $\mathrm{FEV}_{1} \leq 40 \%$ predicted

Plethysmographic RV $\geq 180 \%$ predicted with TLC $>120 \%$ predicted

Peak systolic pulmonary artery pressure $<50 \mathrm{~mm} \mathrm{Hg}$ on color Doppler echocardiography

Arterial carbon dioxide $<50 \mathrm{~mm} \mathrm{Hg}$

Diffusion capacity of carbon monoxide $>20 \%$ predicted

Quit smoking for at least 4 mo

Age $\leq 80$ y

ASA score $\leq 3$

Body mass index $>18<29$.

No instable angina or ventricular arrhythmia

No comorbid condition that would significantly increase operative risk or negatively affect participation in a vigorous respiratory rehabilitation program

No neoplastic disease with life expectancy $<12$ mo

No previous pleurodesis or thoracotomy in the hemithorax targeted for LVRS

\section{Exclusion criteria}

Radiologic evidence of extensive pleural adhesions or previous thoracotomy in hemithorax targeted for LVRS

Patient refusal or noncompliance to thoracic epidural anesthesia and awake surgery

Patient refusal or noncompliance to general anesthesia and 1-lung ventilation

Unfavorable anatomy for thoracic epidural anesthesia

Previous surgery of the cervical or upper thoracic spine

Compromised coagulation with thromboplastin time $<80 \%$, prothrombin time $>40 \mathrm{sec}$, platelet count $<100 / \mathrm{nL}$, or bleeding disorder

$T L C$, Total lung capacity; $A S A$, American Society of Anesthesiology; BMI, body mass index.

TABLE E2. Clinical results in the study groups

\begin{tabular}{|c|c|c|c|c|c|c|c|c|}
\hline & \multicolumn{2}{|c|}{ Baseline } & \multicolumn{2}{|c|}{$6 \mathrm{mo}$} & \multicolumn{2}{|c|}{$12 \mathrm{mo}$} & \multicolumn{2}{|c|}{$24 \mathrm{mo}$} \\
\hline & Awake & Control & Awake & Control & Awake & Control & Awake & Control \\
\hline BMI & $24 \pm 4$ & $23 \pm 3$ & $24 \pm 3$ & $23 \pm 3 \dagger$ & $25 \pm 2 \dagger$ & $24 \pm 2 \dagger$ & $26 \pm 2 \dagger$ & $24 \pm 2 \dagger$ \\
\hline $\mathrm{FEV}_{1}(\mathrm{~L})$ & $0.82 \pm 0.3$ & $0.78 \pm 0.2$ & $1.11 \pm 0.3^{*}$ & $1.06 \pm 0.2^{*}$ & $1.11 \pm 0.2^{*}$ & $1.07 \pm 0.2^{*}$ & $1.02 \pm 0.2^{*}$ & $0.99 \pm 0.2^{*}$ \\
\hline $\mathrm{FEV}_{1}(\%)$ & $29 \pm 9$ & $27 \pm 7$ & $40 \pm 10^{*}$ & $37 \pm 9 *$ & $40 \pm 9 *$ & $37 \pm 9^{*}$ & $36 \pm 9 *$ & $34 \pm 8^{*}$ \\
\hline $\mathrm{FVC}(\mathrm{L})$ & $2.36 \pm 0.8$ & $2.35 \pm 0.5$ & $2.87 \pm 0.6^{*}$ & $2.72 \pm 0.6^{*}$ & $2.84 \pm 0.6^{*}$ & $2.80 \pm 0.5^{*}$ & $2.73 \pm 0.6^{*}$ & $2.76 \pm 0.5^{*}$ \\
\hline $\mathrm{FVC}(\%)$ & $64 \pm 17$ & $63 \pm 16$ & $78 \pm 13^{*}$ & $74 \pm 17^{*}$ & $78 \pm 13 *$ & $76 \pm 16^{*}$ & $73 \pm 13^{*}$ & $75 \pm 13^{*}$ \\
\hline $\mathrm{RV}(\mathrm{L})$ & $5.05 \pm 1.0$ & $5.29 \pm 0.7$ & $4.0 \pm 0.8^{*}$ & $4.37 \pm 0.6^{*}$ & $4.07 \pm 0.9^{*}$ & $4.46 \pm 0.6^{*}$ & $4.29 \pm 0.7^{*}$ & $4.69 \pm 0.6^{*}$ \\
\hline $\mathrm{RV}(\%)$ & $217 \pm 40$ & $229 \pm 38$ & $171 \pm 29^{*}$ & $188 \pm 34^{*}$ & $174 \pm 34^{*}$ & $194 \pm 37^{*}$ & $181 \pm 28^{*}$ & $201 \pm 36^{*}$ \\
\hline TLC (L) & $8.17 \pm 1.5$ & $8.81 \pm 0.9$ & $7.43 \pm 1.5^{*}$ & $8.15 \pm 0.8^{*}$ & $7.40 \pm 1.5^{*}$ & $8.21 \pm 0.8^{*}$ & $7.50 \pm 1.5^{*}$ & $8.38 \pm 0.7 *$ \\
\hline $\operatorname{TLC}(\%)$ & $129 \pm 17$ & $140 \pm 17$ & $117 \pm 17^{*}$ & $129 \pm 13^{*}$ & $117 \pm 17^{*}$ & $130 \pm 14^{*}$ & $119 \pm 16^{*}$ & $132 \pm 14^{*}$ \\
\hline 6-min WT (m) & $300 \pm 112$ & $329 \pm 98$ & $392 \pm 114^{*}$ & $418 \pm 74^{*}$ & $403 \pm 78^{*}$ & $432 \pm 79^{*}$ & $391 \pm 83^{*}$ & $418 \pm 74 *$ \\
\hline MITT (Bruce class) & $0.58 \pm 0.3$ & $0.65 \pm 0.4$ & $1.53 \pm 0.7^{*}$ & $1.54 \pm 0.7 *$ & $1.73 \pm 0.6^{*}$ & $1.82 \pm 0.6^{*}$ & $1.60 \pm 0.6^{*}$ & $1.66 \pm 0.6^{*}$ \\
\hline $\mathrm{PAO}_{2}(\mathrm{~mm} \mathrm{Hg})$ & $68 \pm 8.0$ & $67 \pm 6.0$ & $72 \pm 9.0^{*}$ & $70 \pm 6.0^{*}$ & $71 \pm 9.0 \dagger$ & $70 \pm 5.0^{*}$ & $69 \pm 9.0$ & $68 \pm 6.0$ \\
\hline $\mathrm{PaCO}_{2}(\mathrm{~mm} \mathrm{Hg})$ & $41 \pm 5.0$ & $41 \pm 3.0$ & $39 \pm 3.0 \ddagger$ & $40 \pm 3.3 \ddagger$ & $39 \pm 3.0$ & $40 \pm 2.0 \ddagger$ & $41 \pm 4.0$ & $41 \pm 3.0$ \\
\hline Dyspnea index (score) & $3.5 \pm 0.6$ & $3.4 \pm 0.6$ & $2.14 \pm 0.7^{*}$ & $2.21 \pm 0.7^{*}$ & $2.00 \pm 0.6^{*}$ & $2.12 \pm 0.6^{*}$ & $2.26 \pm 0.8^{*}$ & $2.19 \pm 0.6^{*}$ \\
\hline PF (36-Item Short Form score) & $28 \pm 13$ & $29 \pm 13$ & $59 \pm 17 *$ & $51 \pm 17^{*}$ & $59 \pm 16^{*}$ & $52 \pm 17^{*}$ & $54 \pm 11^{*}$ & $53 \pm 15^{*}$ \\
\hline
\end{tabular}

$B M I$, Body mass index; MITT, maximal incremental treadmill test; dyspnea index, modified Medical Research Council dyspnea score; $T L C$, total lung capacity; $P F$, physical

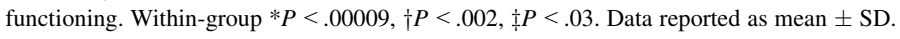

